\title{
Plane-wave based electron tunneling through field emission resonance states
}

\author{
A. Garcia-Lekue, ${ }^{1,2}$ D. Sánchez-Portal, ${ }^{1,3}$ A. Arnau, ${ }^{1,3,4}$ and L. W. Wang ${ }^{5}$ \\ ${ }^{1}$ Donostia International Physics Center (DIPC), Paseo Manuel de Lardizabal 4, E-20018 San Sebastian, Spain \\ ${ }^{2} I K E R B A S Q U E$, Basque Foundation for Science, E-48011 Bilbao, Spain \\ ${ }^{3}$ Centro de Fisica de Materiales CFM - MPC, Centro Mixto CSIC-UPV, Apdo. 1072, San Sebastian, Spain \\ ${ }^{4}$ Dpto. de Fisica de Materiales UPVIEHU, Facultad de Quimica, Apdo. 1072, San Sebastian, Spain \\ ${ }^{5}$ Computational Research Division, Lawrence Berkeley National Laboratory, Berkeley, California 94720, USA
}

(Received 12 July 2013; revised manuscript received 14 October 2013; published 31 October 2013)

\begin{abstract}
Field emission resonances (FERs) on $\mathrm{Cu}(100)$ surface are investigated by means of tunneling regime simulations performed with a plane-wave based transport calculation method. FERs are located near the surface and decay into the vacuum, and their accurate simulation requires a faithful description of vacuum states. This type of simulations is thus not possible using the popular transport methods based on atom-centered localized basis sets and the use of plane waves becomes important. We introduce a procedure to treat self-consistently (SC) the finite bias nonequilibrium problem in tunneling regime. Image potential effects are included in a semiempirical way within the SC calculation. Tunneling through FERs is studied following a practical strategy to approximate the inelastic transmission for states lying in the band gap of the surface. As our approach permits the use of any tip geometry, tip effects on the energy and wave functions of FERs are explored. The method reported here provides an ideal tool for the simulation of FERs aimed at the understanding of experimental STS (scanning tunneling spectroscopy) observations.
\end{abstract}

DOI: 10.1103/PhysRevB.88.155441

PACS number(s): 71.15.-m, 73.63.-b, 73.22.-f

\section{INTRODUCTION}

The investigation of electron processes at surfaces is an extremely active field, since such knowledge permits the understanding of many physical phenomena, such as electron scattering or electron transport. ${ }^{1,2}$ These phenomena are intimately related to the performance of electronic devices and, hence, they are vital in the development of future electronic technology.

Experimentally, scanning tunneling spectroscopy (STS) is a powerful tool to probe the surface electronic structure and electron dynamics. In most cases, STS are recorded at low applied bias voltages, where the influence of the applied electric field is not so relevant. However, STS can also be operated in the field-emission regime, i.e., at bias voltages larger than the tip work function. Under these conditions, STS can be used to investigate image potential states. The image potential is caused by the interaction of electrons with the surface polarization induced by the electron itself, and due to its long-range character, the resulting quantized electronic states form a Rydberg-like series. ${ }^{3-5}$ In field emission-regime, image-potential states are Stark-shifted and become fieldemission resonances (FERs).

In STS experiments, FERs can be detected using $z-V$ spectroscopy, that is, operating the experimental setup in constantcurrent mode. Within this operation mode, the feedback loop is left on and, thus, the tunneling current $I$ is kept constant while scanning the bias voltage $V$. This way, the derivative of $I$ with respect to $V, \partial_{V} I(V)$, as well as the varying tip-sample separation $z(V)$, are acquired simultaneously. Under these experimental conditions, every time a new FER is available for tunneling a jump in $z(V)$ and a peak in $\partial_{V} I(V)$ are observed. ${ }^{6,7}$

Over the past years, a renewed interest on FERs has emerged and many experimental studies have been reported ${ }^{8-19}$ The theoretical interpretation of FERs is commonly done using simple one-dimensional (1D) models for the description of the surface potential. ${ }^{14,20-23}$ However, there are many drawbacks using model effective mass theory with 1D model potentials that can be overcome with an atomicscale description of the effective potential in which electrons propagate: the description of states near the surface accounting for corrugation and, in particular, the different dispersion of surface states from those located in the bulk and vacuum regions. In this work, we describe a density functional theory (DFT) based calculation method that treats the full system under nonzero bias voltage and solves the potential of the tip-sample system self-consistently.

Nevertheless, the well-known shortcoming of DFT to describe the asymptotic behavior of the image potential towards vacuum has prevented its use for the analysis of image states. Different strategies have been proposed to restore the correct imagelike behavior outside the surface. ${ }^{24-26}$ In this work, such DFT limitation has been overcome by applying a matching procedure ${ }^{23,27,28}$ to enforce the correct imagelike potential tail within our DFT-based self-consistent approach. The inclusion of the image potential, together with the self-consistent treatment of the finite voltage drop across the system, enables the quantitative analysis of FERs.

The DFT based theoretical description of the FERs introduced here is based on a quantum transport calculation procedure which solves the scattering states using a plane-wave basis set. ${ }^{29-31}$ From the scattering states, one can straightforwardly calculate the transmission coefficients and the electronic current of the system. As shown in Ref. 32, this plane-wave based method provides a very accurate description of the scattering states in the vacuum region. FERs have a maximum outside the surface of the metal. The higher the order in the Rydberg-like series, the further the maximum of the FER state is from the surface and into the vacuum region. Therefore a precise description of vacuum wave functions is key for a proper treatment of such states. Within this scenario, our plane-wave based method provides a very useful tool to characterize FERs. 
A relevant issue regarding FERs is their dependence on tip properties. FER states are unavoidably Stark-shifted due to the electric field induced by the tip. Thus different tips can provide significantly distinct FER spectra. ${ }^{15,16}$ Clearly, this tip effect is specially relevant in the case of FERs with $n>1$, as they are located closer to the tip. Nevertheless, since the tip properties are very difficult to control in the experiments, the analysis of higher $n$ states poses serious difficulties, and the discussion of experimental observations is often restricted to the $n=1$ FER state. ${ }^{15}$ Most of previous descriptions of FERs have been based on the use of $1 \mathrm{D}$ model potentials that restrict the treatment to planar tip geometry only. ${ }^{14,16,23,33,34}$ With our approach, instead, any tip geometry can be used, which opens up the possibility of understanding the influence of different tips on FERs.

With the aim of demonstrating the potential of our method, we study a well-established system, the $\mathrm{Cu}(100)$ surface. On this surface, a projected band gap exists around $\bar{\Gamma}$ point, which together with the tip-field effect gives rise to FERs. . $^{14,16,19,35,36}$ The influence of the electric field, fixed by the applied voltage and surface-tip distance, is investigated. We also study tunneling through FERs and we introduce a procedure to calculate the inelastic transmission for the FERs lying in the band gap of the $\bar{\Gamma}$ point electronic structure of the receiving electrode into the lower energy electrode states. Inelastic processes might occur, for example, via phonon emission or electron-electron interaction. As a first attempt, the tip shape has been approximated by a flat surface. Next, the influence of the detailed atomic structure of the tip is investigated by including an atomically sharp tip.

The paper is organized as follows. In Sec. II A, we describe the computational method used for the transport calculation through FERs, including a brief description of the formalism introduced in Refs. 29 and 30. We also introduce the procedures employed to incorporate the image potential and simulate inelastic transmission. In Sec. III, the main results of our calculations for a $\mathrm{Cu}(100)$ model system are presented and discussed. Concluding remarks are summarized in Sec. IV.

\section{CALCUlation METHOD}

\section{A. Transport method for finite bias voltage}

For the calculation of the scattering states, we consider the system as sketched in Fig. 1(a), consisting of a central part connected to bulk semi-infinite left and right electrodes. The

(a)

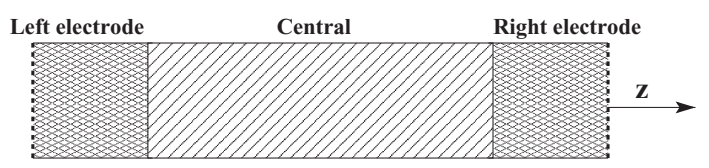

(b)

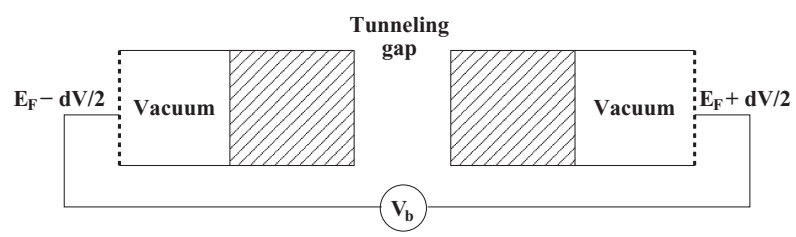

FIG. 1. (a) Full model system used in the calculation method described in this work. (b) Modified central region used for the SC calculation of the potential. current across the system is given by

$$
I=\frac{2 e}{h} \int_{\mu_{L}}^{\mu_{R}} T(E) d E,
$$

where $\mu_{L}$ and $\mu_{R}$ are the left and right electrode chemical potentials, assuming that the current flows from right to left. $T(E)$ is obtained from the scattering states of the full system of Fig. 1(a), which are the solution of the following Schrödinger equation:

$$
H \psi_{\mathrm{sc}}(r)=E \psi_{\mathrm{sc}}(r),
$$

where $H=\left[-\frac{1}{2} \nabla^{2}+V(r)\right]$ is the single-particle Hamiltonian, and satisfying the corresponding scattering state boundary conditions. In order to solve Eq. (2), auxiliary periodic boundary conditions are imposed on the system, which turns the open boundary problem into a conventional closed boundary problem. Details of the method can be found in Refs. 29 and 30.

For large voltages, the coupled charge/potential problem must be solved self-consistently, in order to adequately describe the nonequilibrium state of the system. Previously, we introduced a self-consistent (SC) algorithm for the calculation of the system potential under finite bias voltage. ${ }^{37}$ In that method, the self-consistent DFT potential for zero applied bias is calculated for the central region of Fig. 1(a). Then, to include the effect of the external bias voltage, an initial guess for the potential in the central region is built based on a sinelike potential. This central potential is matched to left and right bulk electrode potentials shifted by $-V_{b} / 2$ and $+V_{b} / 2$, respectively, where $V_{b}$ represents the applied voltage. Taking the potential for the full system constructed this way as an initial guess, a SC iteration is carried out by occupying the calculated scattering states by the left and right electrode Fermi energies to yield the charge density, and solving the Poisson equation satisfying the left and right electrode potential boundary conditions. ${ }^{37}$ Finally, the scattering states calculated with the converged potential yield the final transmission coefficients. Although accurate, such a SC can be computationally expensive depending on the size of the system. For this reason, we have implemented a novel approximated SC calculation approach to simulate the nonequilibrium system.

Within the new approach, we first calculate the selfconsistent potential of the central part. This is done by employing a modified central region surrounded by vacuum on left and right sides, as displayed in Fig. 1(b). The effect of the applied bias is taken into account by redefining the occupation of the central system in the following way:

$$
\begin{aligned}
\rho_{C}(\mathbf{r})= & \sum_{i}\left[\left|\psi_{i}(\mathbf{r})\right|^{2} W_{L}(\mathbf{r}) f\left(\epsilon_{i}-\mu_{L}\right)\right. \\
& \left.+\left|\psi_{i}(\mathbf{r})\right|^{2} W_{R}(\mathbf{r}) f\left(\epsilon_{i}-\mu_{R}\right)\right],
\end{aligned}
$$

where $\mu_{L(R)}$ are the left and right Fermi energies given by $E_{F} \pm V_{b} / 2$, respectively, and $f\left(\epsilon_{i}-\mu_{L(R)}\right)$ are the corresponding Fermi-Dirac distributions. $W_{L(R)}(\mathbf{r})$ are some auxiliary mask functions, and $W_{R}=1-W_{L}$.

This procedure works well for tunneling cases, like in our FER study, where the charge density at the barrier region is very small. In such cases, the $W_{L}(\mathbf{r})$ is one at the left electrode, then drops abruptly in the barrier region (at the 
point of maximum potential, or said minimum charge density) to zero, and it remains to be zero at the right electrode. Reference 38 shows that the charge density obtained in that way is almost indistinguishable to the charge density obtained by occupying the scattering states as described in Ref. 30. As will be described in Sec. IIC, in our SC calculation of the biased central region, we have also included an image potential (as an external fixed potential) on top of the local density approximation (LDA) potential, to correct the LDA error in the exchange and correlation potential.

Once the SC potential for the central region is obtained following the new occupation procedure, it is matched to left and right electrode potentials. From the resulting full system potential, the scattering states are calculated following the approach of Refs. 29 and 30. In this way, the scattering states of the full system under bias voltage are achieved in a SC manner.

\section{B. Image potential}

An appropriate description of the FERs requires to include the correct imagelike behavior outside the surface in the SC calculation of the potential for the central region. For this purpose, we have developed the procedure described in the following and schematically depicted in Fig. 2.

First, we consider a single surface and we calculate the corresponding DFT potential $V_{\text {DFT }}$ under zero applied bias. The potential in the vacuum region $V_{\text {vac }}$ is given by $V_{\mathrm{DFT}}$ far away from the surface. Then, the image potential outside this surface can be expressed as

$$
V_{\mathrm{im}}(z)=V_{\mathrm{vac}}-\frac{1}{4\left|z-z_{\mathrm{im}}\right|} .
$$

$z_{\text {im }}$ represents the position of the image plane and is a parameter, which can be determined from the center of the static screening charge. ${ }^{39}$

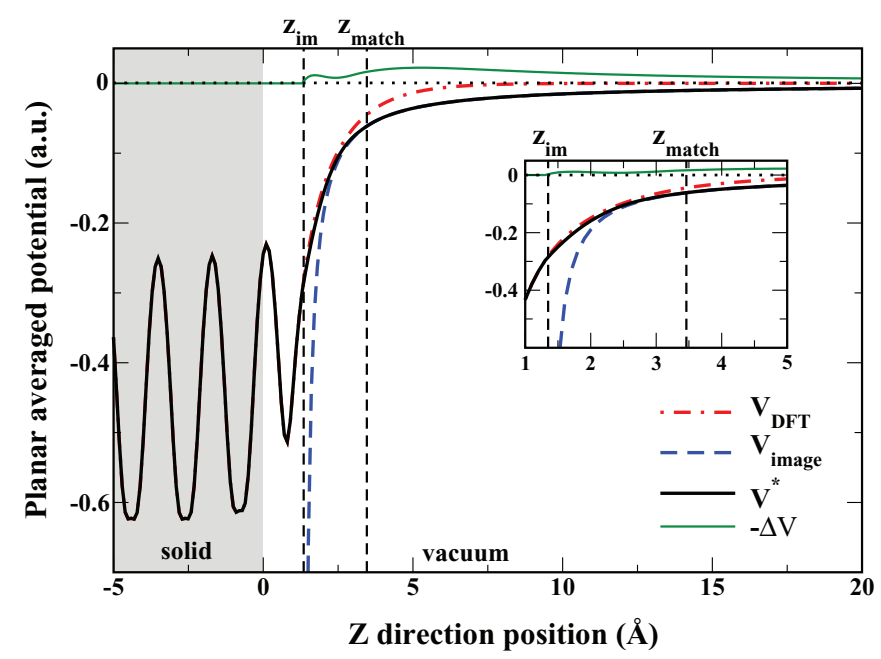

FIG. 2. (Color online) Planar averaged potential for $\mathrm{Cu}(100)$ surface. $V^{*}(z)$ (black/thick solid) is obtained by connecting the planar averaged DFT potential $V_{\mathrm{DFT}}(z)$ (red/dashed dotted) at $z_{\text {im }}$ to the image potential (blue/dashed) at $z_{\text {match }} . \Delta \mathrm{V}$ (green/thin solid) gives the difference between $V^{*}$ and DFT potentials. The zero of energy is at the vacuum level. The gray area represents the solid system that extends towards the left.
Next, using the DFT and image potentials introduced above, the following modified potential is built:

$$
V^{*}(z)= \begin{cases}V_{\mathrm{DFT}}(z) & \text { if } \quad z \leqslant z_{\mathrm{im}}, \\ V_{\mathrm{im}}(z) & \text { if } \quad z \geqslant z_{\text {match }} .\end{cases}
$$

Across the matching region $\left[z_{\mathrm{im}}, z_{\text {match }}\right]$, the $V^{*}(z)$ potential is smoothly transformed from the DFT potential at $z_{\text {im }}$ to the imagelike potential at $z_{\text {match }}$, as follows:

$$
V^{*}(z)=V_{\mathrm{DFT}}(z) f(z)+V_{\mathrm{im}}(z)[1-f(z)],
$$

where $f(z)$ is defined as

$$
f(z)=\cos ^{2}\left[\left(z-z_{\text {im }}\right) \pi / 2\left(z_{\text {match }}-z_{\text {im }}\right)\right],
$$

in the same fashion as in Ref. 23. The final $V^{*}(z)$ potential is represented by a solid line in Fig. 2.

Finally, $\Delta V(z)$ is defined as the difference between the modified potential and the planar averaged DFT potential at zero bias, i.e., $\Delta V(z)=V^{*}(z)-V_{\mathrm{DFT}}(z)$. We observe from Fig. 2 that this function is strictly zero inside the surface, reaches its maximum magnitude near the surface in the vacuum side and decays to zero far away from the surface into the vacuum. $\Delta V(z)$ is first calculated in the zero-bias calculation, then added as a fixed external potential in the single-particle Hamiltonian when solving the SC potential of the finite bias problem described in Sec. II A. This procedure ensures a correct imagelike behavior of the final SC potential in the vacuum region. Based on our self-consistent calculation, we found that the changes on the surface charge density under the different applied bias voltages considered in our study are rather small. Thus we have used the same image plane and image potential for all the applied bias voltages.

Our procedure would allow the fitting of energy levels to their corresponding experimental values, providing a way to determine the model parameters $z_{\text {im }}$ and $z_{\text {match }}$, which describe the metal surface properties. However, as in this work our main objective is the description of the calculation method and its capabilities, we employ a minimal matching region scheme and the position of the image plane reported previously by Chulkov et al. ${ }^{40}$

It should be mentioned here that, as our main interest lies on the analysis of the FERs belonging to the sample surface, we have only included the image potential on that side of the tunneling gap. For the high bias voltages used in this work, the FERs will be very localized near the surface region. In this state localization region, and due to the large tip-sample distances we employ, the image potential of the tip will be small and rather flat. ${ }^{41}$ Hence neglecting the tip image potential will only introduce a minor energy shift in the energy of the FERs. Furthermore, the image potential from the tip might sensitively depend on the shape of the tip in a way which is difficult to include in a simple model. All in all, including this relatively small image potential might complicate our analysis and, consequently, it has been neglected in our simulations.

\section{Transmission through field emission resonances}

As mentioned above, once the scattering states are known the transmission coefficient can be calculated in a straightforward way. This transmission coefficient accounts for elastic processes, where electrode states incoming from the right 
with energy $E$ are transmitted onto electrode states of the left side with energy $E$. Within this scenario, there would not be any transmission through FERs, as they lie inside the energy gap of the $\bar{\Gamma}$ point electronic band structure. However, inelastic processes of the incoming electrons with the surface and also with the bulk states yield a nonzero transmission for the FERs. These can be taken into account empirically by including an imaginary potential $V_{i},{ }^{14,19,42,43}$ which is treated as a phenomenological parameter accounting for inelastic effects. $V_{i}$ exists only inside the left surface and extends into the left electrode. If the imaginary potential is treated as a perturbation potential, the current through the FERs due to inelastic effects can be written as

$$
I_{\text {inel }}=\frac{e}{h} \int V_{i}(r)\left|\psi_{\mathrm{sc}}(r)\right|^{2} d r
$$

where $\psi_{\text {sc }}(r)$ is the scattering state obtained from Eq. (2) using the unperturbed Hamiltonian $H$. This approximation is appropriate when $V_{i}$ does not change the FER significantly, which we assume to be the case here. In principle, one could choose a value of $V_{i}$ such that the experimental lifetimes of image states are reproduced. Here, we employ a fixed value of $V_{i}$, as given in previous works. ${ }^{14}$ The use of a constant imaginary potential is based on the so-called penetration approach, which assumes that the lifetime of an image state is proportional to the overlap of the probability density with the metal substrate. ${ }^{44,45}$ Neglecting the energy dependence of the imaginary potential should not qualitatively change our conclusions.

From the knowledge of the inelastic current, we can calculate the inelastic transmission. In our case, the inelastic current $I_{\text {inel }}$ is always much smaller than the current $I_{\text {incoming }}$ from the incoming wave of the scattering state. Note that $I_{\text {incoming }}$ is not the elastically transmitted current from the right electrode to the left, rather it is the current of the incoming electrode state inside $\psi_{\mathrm{sc}}$, which in our case has been reflected back completely to the right electrode under the elastic approximation. In this case, the total transmission due to inelastic scattering is simply

$$
T_{\text {inel }}=\frac{I_{\text {inel }}}{I_{\text {incoming }}} .
$$

In summary, the calculation of the tunneling transmission across FERs is performed using a DFT based procedure to obtain the SC potential for the full system under bias voltage including image potential effects. Then, this SC potential is used to calculate the corresponding scattering states $\psi_{\mathrm{sc}}$. Finally, from the knowledge of $\psi_{\mathrm{sc}}$ and including an imaginary potential $V_{i}$ to account for inelastic effects, the inelastic transmission through FERs is achieved.

\section{RESULTS}

As mentioned in $\mathrm{Sec} . \mathrm{I}, \mathrm{Cu}(100)$ is a well-characterized surface, which has been theoretically and experimentally investigated for its FERs. ${ }^{14-16,19}$ Therefore we believe it is an ideal candidate to test our procedure of the tunneling through FERs. Figure 3(a) shows an atomistic illustration of the model set up used in this work, which consists of two facing $(3 \times 3)-\mathrm{Cu}(100)$ surfaces. Calculations are performed using norm conserving pseudopotentials and local-density
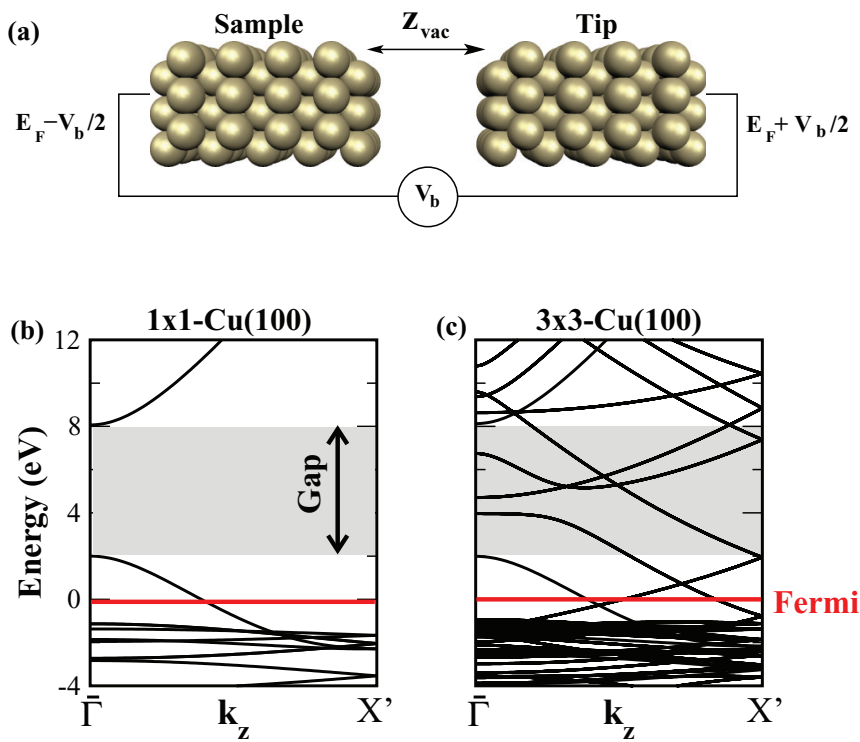

FIG. 3. (Color online) (a) Atomistic illustration of the two-facing $(3 \times 3)-\mathrm{Cu}(100)$ surfaces used in the calculations. (b) and (c) The band structure of $(1 \times 1)-\mathrm{Cu}(100)$ and $(3 \times 3)-\mathrm{Cu}(100)$ surface, respectively, in the $\bar{\Gamma} X$ direction, where $k_{z}$ is the momentum in the direction of propagation, i.e., perpendicular to the $x y$ surface.

approximation (LDA). For the electrode and for the central region under finite bias, the calculations are converged with respect to the number of $k$ points. For the scattering state calculations, only the $\bar{\Gamma}$ point in the $(x, y)$ directions of the supercell is included.

Note, our $\bar{\Gamma}$ point is the $\bar{\Gamma}$ point of the $3 \times 3$ supercell, thus it already includes the $3 \times 3\left(k_{x}, k_{y}\right)$ points of the primary cell in our calculation. The band structure of the $1 \times 1$ primary cell electrode at $\bar{\Gamma}^{\prime}$ point is plotted in Fig. 3(b). As we can see, there is a $\sim 6 \mathrm{eV}$ band gap, which provides the possibility for the existence of surface states. On the other hand, as illustrated in Fig. 3(c), the $\bar{\Gamma}$ point band structure of the $3 \times 3$ supercell electrode does not show any band gap, due to the band folding in the $x$ and $y$ directions. This is consistent with our observation that, in practice, all the observed surface states of the $3 \times 3$ supercell come from the $\bar{\Gamma}^{\prime}$ point of the $1 \times 1$ primary cell.

\section{A. Field emission resonance states}

In constant current mode, the tip displacement is (on average) approximately proportional to the applied bias voltage, except at particular values where the resonance appears and steps in $z(V)$ are observed [correspondingly, peaks in $\left.\partial_{V} I(V)\right]$. This global behavior can be mimicked by using a linear $z(V)$ ramp that essentially corresponds to a constant field $(V / z)$ at the tunnel junction.

In this work, we have reproduced the experimental strategy by investigating the field-emission resonance states for the following $\left(z_{\mathrm{vac}}, V_{b}\right)$ set points (see Fig. 4): $(9.6 \AA, 5.0 \mathrm{~V}),(13.2 \AA$, $6.7 \mathrm{~V})$, and $(16.8 \AA, 8.4 \mathrm{~V})$. These set points correspond to a field of $\sim 0.5 \mathrm{~V} / \AA$, which is slightly larger but still of the order of the field values involved in STS experimental observations. Therefore, despite the approximations contained in our model, it should capture the main physical ingredients of the problem under investigation. 


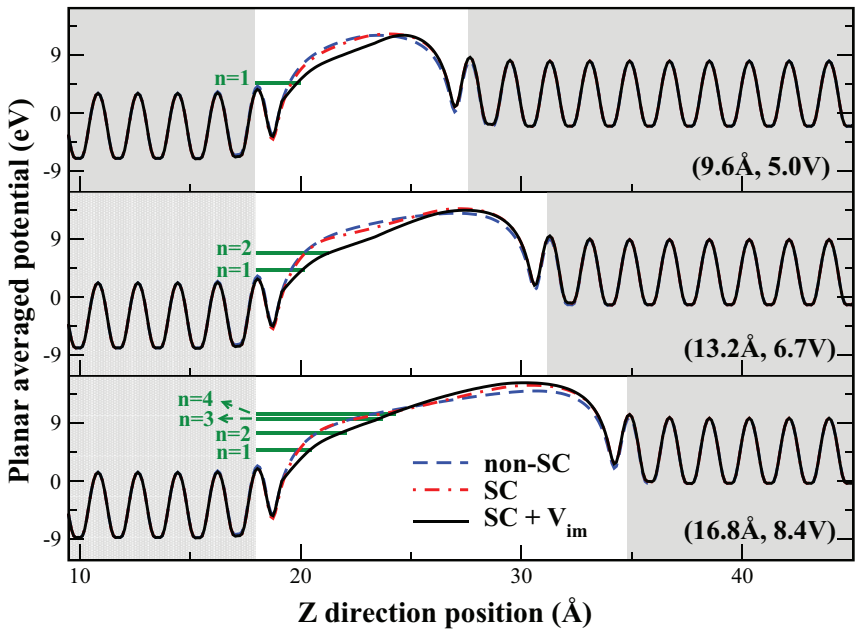

FIG. 4. (Color online) Full system potential for three different $\left(z_{\mathrm{vac}}, V_{b}\right)$ set points, as obtained non-self-consistently without image potential (blue, dashed line), self-consistently without image potential (red, dash-dotted line), and self-consistently including image potential (black, solid line). The gray area represents $\mathrm{Cu}(100)$ metallic region. The position of the FERs at $\bar{\Gamma}$ is also indicated (green, horizontal solid lines). The zero of energy is at the Fermi level of the left electrode.

In first place, we have tested the adequacy of our procedure for the SC calculation of the system potential under applied bias voltage. In Fig. 4, we compare the SC and non-SC potentials corresponding to the set points given above. The non-SC potential is obtained employing a sinelike potential for the central region, as explained in Sec. II A. Given the size of the system under investigation here and the large values of the voltages needed for the simulation of the FERs, occupying scattering states to calculate the SC potential as in our previous work ${ }^{37}$ would be computationally very expensive. From Fig. 4, it can be seen that the SC potential provides a larger bump and barrier to the potential near the right electrode. Besides, as expected, the field is very efficiently screened by the metal leads and the average potential inside both leads is flat almost up to the surface.

These potential differences between SC and non-SC calculations seem small. In fact, the energies of the first four FERs for the (16.8 $\AA, 8.4 \mathrm{~V})$ summarized in Table $\mathrm{I},{ }^{46}$ do not exhibit a remarkable influence of the self-consistency calculation. However, the small differences between non-SC and SC potentials can be important regarding confinement

TABLE I. Energy values in $\mathrm{eV}$ of the first four FERs of $\mathrm{Cu}(100)$ corresponding to the $(16.8 \AA, 8.4 \mathrm{~V})$ set point, as obtained nonself-consistently (non-SC), or self-consistently with $\left(\mathrm{SC}+V_{\mathrm{im}}\right)$ and without (SC) image potential. These energy values are measured with respect to the Fermi level of the left electrode $\left(\mu_{L}\right)$.

\begin{tabular}{lccc}
\hline \hline & $E($ non-SC) & $E(\mathrm{SC})$ & $E\left(\mathrm{SC}+V_{\mathrm{im}}\right)$ \\
\hline$n=1$ & 6.16 & 6.02 & 4.81 \\
$n=2$ & 8.05 & 7.96 & 7.51 \\
$n=3$ & 9.76 & 9.79 & 9.73 \\
$n=4$ & 10.36 & 10.49 & 10.50 \\
\hline \hline
\end{tabular}

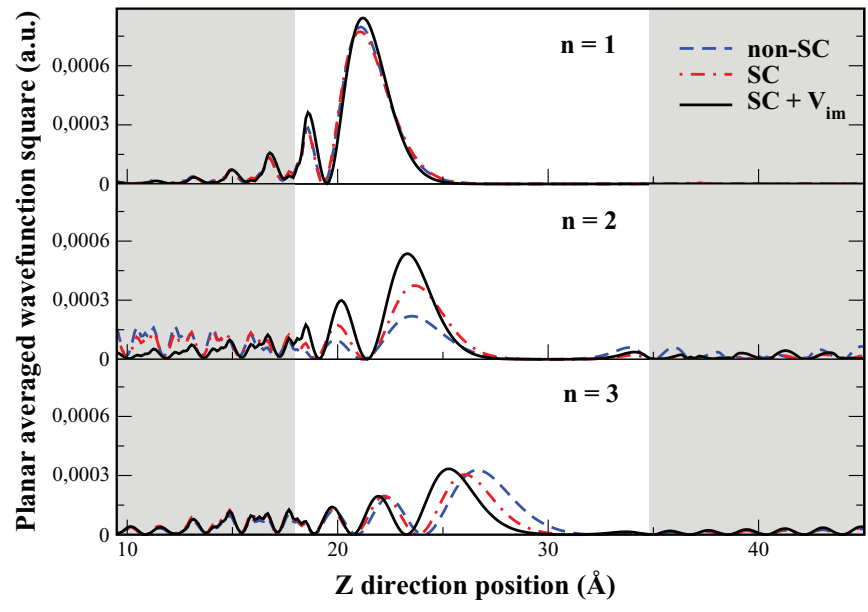

FIG. 5. (Color online) First three FER states of left $\mathrm{Cu}(100)$ surface for ( $16.8 \AA, 8.4 \mathrm{~V})$, as obtained non-self-consistently without image potential (blue, dashed line), self-consistently without image potential (red, dash-dotted line), and self-consistently including image potential (black, solid line). The gray area represents $\mathrm{Cu}(100)$ metallic region.

of image states with high quantum number. The later extend further into the vacuum and, thus, are more sensitive to the exact shape of the barrier near the right electrode, i.e. near the tip in STM experiments. This is illustrated in Fig. 5, where we have represented the wave functions of the first three FER states at $\bar{\Gamma}$ for $(16.8 \AA, 8.4 \mathrm{~V})$ as obtained non-self-consistently and self-consistently. ${ }^{46}$ We observe that the effect of self-consistency on the confinement of FERs is more relevant for states with quantum numbers $n>1$. In particular, we observe that (i) for $n=2$, the amplitude of the non-SC planar averaged density is significantly lower than the $\mathrm{SC}$ result, which is due to the fact that this state is right below the bottom of the conduction band and, thus, tiny modifications of its energy give rise to large differences in the degree of localization; and (ii) for $n=3$, the amplitude of the density is similar but the state is more extended towards the right electrode in the non-SC case.

We have also studied the effect of the image potential based on the comparison of the SC potentials as obtained with and without taking into account the imagelike behavior of the potential on the vacuum side. Within our model system, the left and right $\mathrm{Cu}(100)$ surfaces resemble the sample and tip of the STM set up, respectively. Therefore, since our aim is to investigate the sample FERs, we have only incorporated the image potential on the left $\mathrm{Cu}(100)$ surface, that is, on the sample side. The corresponding full system potential is shown in Fig. 4. The SC potential obtained without image potential would also yield FERs, originated just by the tip induced potential. ${ }^{14}$ However, the energetics of the FERs as well as their confinement will be modified when incorporating the image potential, as one can expect from the variation in the SC potential observed in Fig. 4.

Regarding the effect of the image potential on the energetics of FERs, this is expected to be more significant for FERs with lower quantum numbers (smaller $n$ values). FERs with higher quantum numbers will be dominated by the tip-induced electric field instead and, hence, will show weaker dependence on the 
image potential effects. For the sake of confirming that the role of the image potential is less relevant for higher quantum numbers, we have compared the energies of the FERs for the (16.8 $\AA, 8.4 \mathrm{~V})$ set point as obtained using self-consistently with and without image potential. The results for the first four FERs for the $(16.8 \AA, 8.4 \mathrm{~V})$ are summarized in Table I. As predicted, the effect of the image potential is crucial for the energy of the first FER $(n=1)$ and is still significant for the second FER $(n=2)$. For higher quantum numbers $(n=3,4)$, the role of the image potential in the energy of the FERs is almost negligible $(\sim 1 \%)$.

On the contrary, the effect of the image potential on the wave function of FERs is stronger for states with high quantum number. This is confirmed by the FER states given in Fig. 5, which show the importance of the image tail in determining the spatial distribution of the states lying at high energies and, therefore, more delocalized into the vacuum region. Whereas the image potential does not modify significantly the $n=1$ state [see Fig. 5(a)], the $n=3$ state [see Fig. 5(c)] is trapped closer to the sample surface under the influence of the image potential.

All in all, after having investigated in detail the effect of a self-consistent treatment of the nonequilibrium problem and the role of the image potential, we believe that both ingredients are desirable for a proper description of FERs. Consequently, all the results reported in the following will correspond to calculations done self-consistently and including the image potential on the sample side.

In Fig. 6, we have represented the FERs corresponding to the set points considered in this work, which have been chosen to mimic the experimental mode used for the observation of such states. Accordingly, our first set point $(9.6 \AA$, $5.0 \mathrm{~V})$ allows us to reproduce the $n=1$ FER, which is located very close to the left surface and has an energy of $4.62 \mathrm{eV}$ with respect to the sample Fermi level [see Fig. 6(a)]. ${ }^{16,47,48}$ Then, increasing both the vacuum gap and the bias voltage, i.e., using the $(13.2 \AA, 6.7 \mathrm{~V})$ set point, we are able to identify both $n=1$ and 2 FERs. As seen from Fig. 6(b), the second FER is located further from the left $\mathrm{Cu}(100)$ surface and its energy lies significantly higher, at about $6.89 \mathrm{eV}$ from the left Fermi level. Finally, we have looked at the FERs for the third set point, (16.8 $\AA, 8.4 \mathrm{~V})$. In this case, as shown in Fig. 6(c), $n=3$ and 4 FERs are also available, the maximum of their density being located notably further from the left $\mathrm{Cu}(100)$ surface. However, whereas $n=1$ and 2 FERs decay smoothly into the left electrode, the higher quantum numbers do not exhibit a decaying behavior. This is due to the fact that the energy of these states, 9.73 and $10.5 \mathrm{eV}$ for $n=3$ and 4 respectively, lies above the energy gap of the bulk $\mathrm{Cu}$ at the $\bar{\Gamma}$ point. Hence these FER states couple to bulk electrode states and are referred to as resonant local states.

\section{B. Inelastic transport}

We now turn our attention to the band structure and explore in more detail the coupling of the FERs with electrode states in the leads, which determines the electronic transport. With this aim, the energies of the FERs for the (9.6, $5.0 \mathrm{~V})$ and (13.2 $\AA$, $6.8 \mathrm{~V})$ set points are aligned with the band structures of the left (sample) and right (tip) electrodes in Fig. 7. The shaded
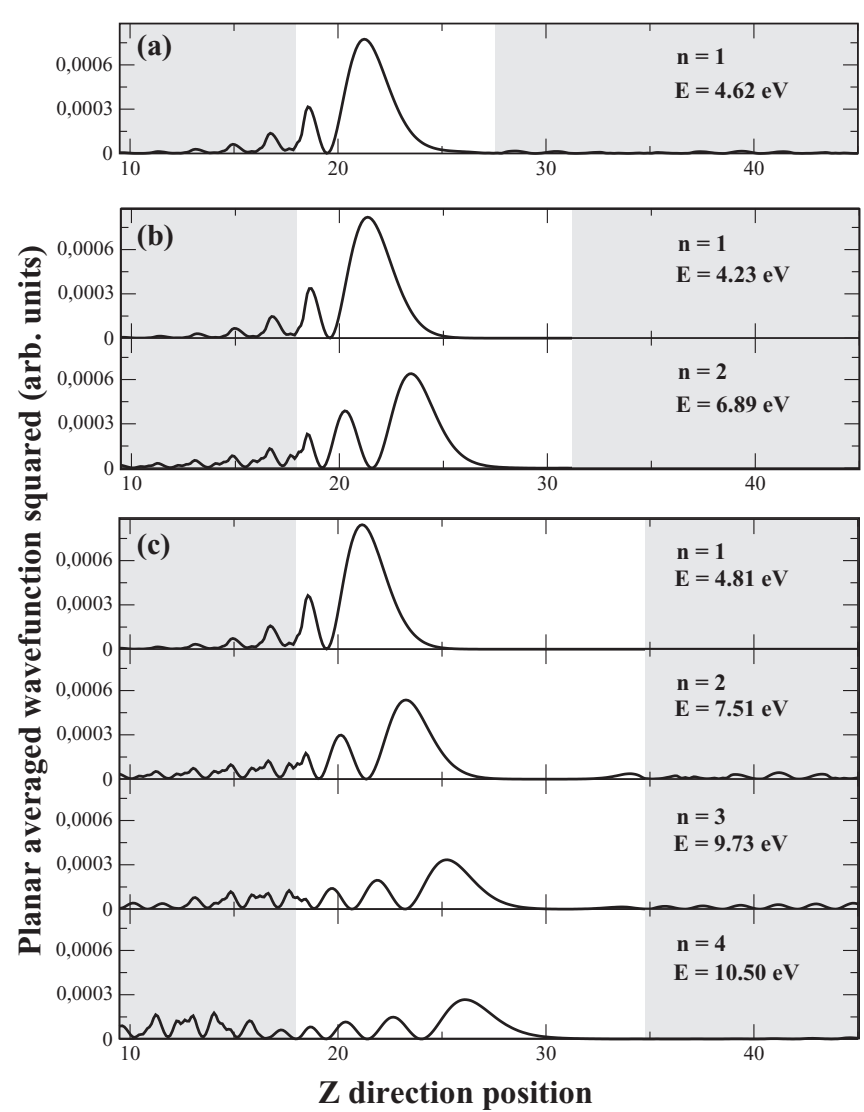

FIG. 6. FER states of left $\mathrm{Cu}(100)$ surface, for three different set points: (9.6 $\AA, 5.0 \mathrm{~V})$ (top), (13.2 $\AA, 6.8 \mathrm{~V})$ (middle), and (16.8 $\AA$, $8.4 \mathrm{~V})$ (bottom). The gray area represents the region over which $\mathrm{Cu}(100)$ extends.

areas represent the energy gap of the bulk band structure of $\mathrm{Cu}$ surface at the $\bar{\Gamma}$ point. As mentioned before, electrode states within this gap (solid black lines inside the shaded gray area) in Fig. 7 appear due to the folding of the band states
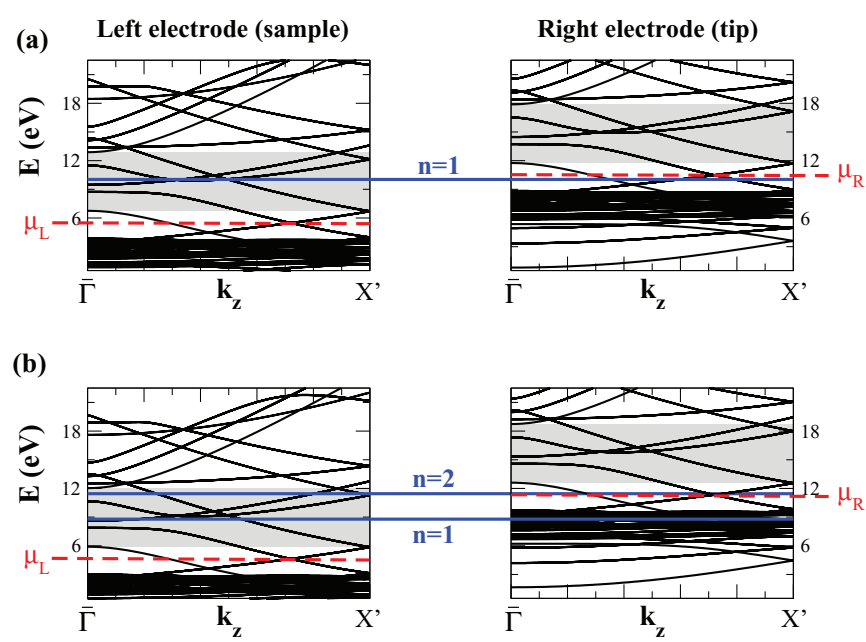

FIG. 7. (Color online) The band alignment between the left and right electrode band structures for the $(9.6 \AA, 5.0 \mathrm{~V})$ (top) and (13.2 , $6.8 \mathrm{~V}$ ) (bottom) set points. Dashed lines represent the Fermi levels of the electrodes, whereas solid lines correspond to the energies of FERs. 
in a $(3 \times 3)-\mathrm{Cu}(100)$ supercell in the calculations. However, with the flat tip, these additional states do not couple to the FERs in Fig. 6 since they belong to the $\bar{\Gamma}$ ' point of the unit cell. In the upper panel, we observe that for $(9.6 \AA$, $5.0 \mathrm{~V})$ only $n=1$ FER exists within the gap of the left electrode and between the Fermi levels of left and right electrodes. For the (13.2 $\AA, 6.8 \mathrm{~V}$ ), shown in the lower panel, the first two FERs are lying inside the gap and between left and right Fermi levels. From these band alignments, we can also extract information on the transmission properties of the system. For example, an incoming electrode state from the right might inelastically tunnel through FER states of the left surface into the left electrode. For the chosen cases, incoming electrode states from the right electrode lying below the right Fermi energy match the energy of the FERs, thus can tunnel through them, and be scattered into the left electrode states via inelastic processes. In particular, the tip electrode states of $(9.6 \AA, 5.0 \mathrm{~V})$ and $(13.2 \AA$, $6.8 \mathrm{~V}$ ) tunnel through $n=1$ and 2 FERs, respectively. For such states, the inelastic transmission can be calculated as described in Sec. II C.

Here, we have calculated the inelastic transmission as a function of the energy using an imaginary potential of $200 \mathrm{meV},{ }^{14}$ which starts leftwards from one unit cell below the $\mathrm{Cu}(100)$ surface in the left electrode. When the imaginary potential is included, inelastic transmission through FER states lying within the energy gap of bulk $\mathrm{Cu}$ can occur. Hence, every time the tip Fermi level $\left(\mu_{R}\right)$ is close to the energy of a FER (see Fig. 6), such state becomes available for tunneling and the transmission is strongly increased. This situation will be reflected as a sharp peak in the inelastic transmission at the position of each FER.

In order to be able to compare our simulated transport properties with experimentally measured FERs, one would need to calculate the inelastic transmission for a large number of bias voltage values given the sharp resonant feature of FER. However, as such a procedure would be computationally very demanding, we have pursued an alternative way to obtain the transmission curve relevant to experiments. Specifically, we have first calculated the inelastic transmission curves as functions of scattering state energy $E$ for the three set points considered in this work. Based on these results, the transmission curve at any bias voltage and at any scattering state energy can be constructed using interpolation techniques. Finally, since we are interested in what occurs at the tip Fermi level, for each bias voltage value we have evaluated the transmission at $\mu_{R}$. The corresponding transmission curve is shown in Fig. 8. The low and high lying energy peaks in Fig. 8 correspond to $n=1$ and 2 FERs, respectively. We have focused on $n=1$ and 2, which are the FER states most sensitive to the surface potential of the sample and, thus, carry essential information regarding its surface electronic structure. ${ }^{9,49}$ Higher lying FERs lie further from the surface and have a stronger influence by the tip. The transmission results shown in Fig. 8 can only be obtained from scattering state calculations. The width of the transmission peak is related to how strong the FER state couple with the incoming electrode from the right electrode.

As described earlier, the $\left(V_{b}, z_{\mathrm{vac}}\right)$ values used for the calculation of the inelastic transmission spectrum shown in Fig. 8 have been chosen as to provide an approximately

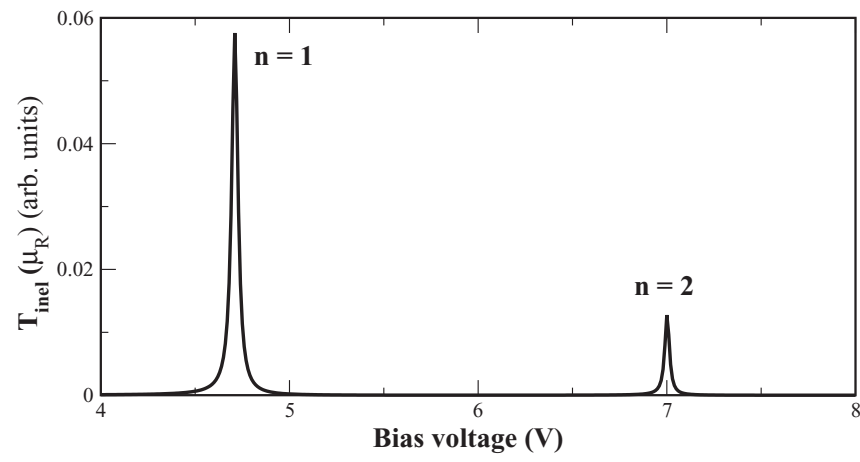

FIG. 8. Inelastic transmission through $n=1$ and 2 FERs.

constant electric field scenario. Equally, when the STS set up is operated in constant current mode, the electric field in the tipsample system is approximately constant. As a consequence, the corresponding differential conductance spectrum $\partial_{V} I(V)$ closely follows the inelastic transmission spectrum evaluated at the tip Fermi level. ${ }^{50}$ Therefore our simulated inelastic transmission spectra, as given in Fig. 8, can, in principle, be compared with experimental conductance spectra of FERs.

\section{Tip effects}

In the following, the influence of the atomic structure of the tip on FERs has been studied. In particular, we have examined how the FERs of $\mathrm{Cu}(100)$ change when a sharp tip is employed, as opposed to the flat tip considered above. As shown in Fig. 9(a), we have modeled an atomically sharp tip by a five-atom pyramid consisting of four basal atoms and one apex atom. This pyramidal tip is periodically repeated in the plane

\section{(a)}



(b)

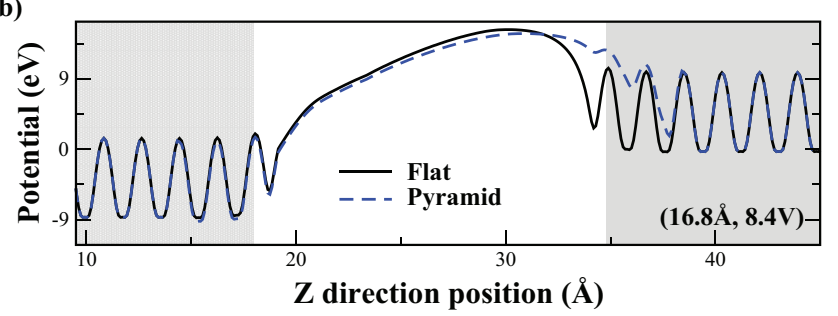

(c)

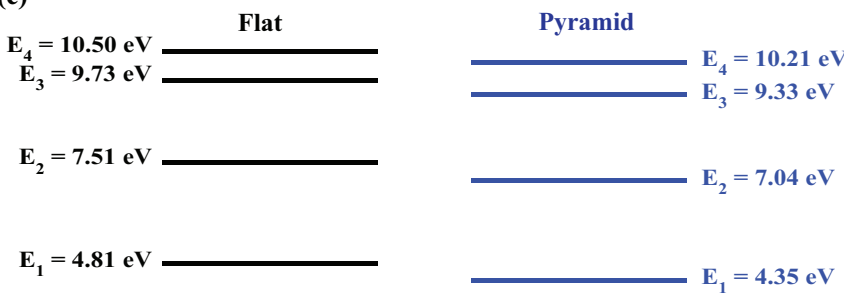

FIG. 9. (Color online) (a) Atomistic illustration of the tunneling junctions with flat (left) and sharp (right) tips. (b) Planar averaged full system potential for (16.8 $\AA, 8.4 \mathrm{~V})$, as obtained using a flat (black, solid line) or sharp (blue, dashed line) tip. (c) Energy of the first four FERs for the junction with flat (left) or sharp (right) tip. The zero of energy in (b) and (c) is at the Fermi level of the left electrode. 




FIG. 10. (Color online) FER states (planar averaged) of left $\mathrm{Cu}(100)$ surface for (16.8 $\AA, 8.4 \mathrm{~V}$ ), as obtained using a flat (black, solid line) or sharp (blue, dashed line) tip.

parallel to the $(3 \times 3)-\mathrm{Cu}(100)$ surface, so tips from adjacent unit cells are more than $7 \AA$ away. In both flat and sharp tip cases, the (16.8 $\AA, 8.4 \mathrm{~V})$ set point has been used for the simulations, where the tunneling distance is measured from the most protruding tip atom to the left $\mathrm{Cu}(100)$ surface (sample). Given the distinct shape of our tips, using the same set point implies that the current flowing across the tunnel junctions of Fig. 9(a) is different. However, FERs are known to show a stronger dependence on the tip properties than on the current at which the FER spectra are taken. ${ }^{16}$ Therefore, despite using slightly different tunneling conditions, our results will permit to extract valuable information about tip effects on FERs, which can be of use for the understanding of experimental STS spectra.

The full system potential (averaged along the $x y$ plane), as obtained with a flat or a sharp tip, is represented in Fig. 9(b). The potential near the tip is, as expected, very different for both tips. However, it quickly decays to the bulk potential, i.e., surface effects in the tip region are rapidly screened towards the right electrode. Clearly, the potential near the sample surface is also noticeably modified by the use of a tip with different atomic structure. Mainly, the potential is widened (i.e., we have a slightly smaller electric field in the junction) in the case of the pyramidal tip, which causes the change of FER energies shown in Fig. 9(c). We observe that, when a sharp tip is employed, FER states are clearly shifted downwards in energy. Besides, whereas the first three FERs shift almost rigidly by $\sim 0.48 \mathrm{eV}$, the energy shift is significantly smaller for $n=4$ $(\sim 0.29 \mathrm{eV})$.
The tip dependence of the FER states can be seen in Fig. 10. The first FER $(n=1)$ is independent of the tip atomic structure. Higher FER states, instead, are clearly affected by the tip, and both their amplitude as well as their decay length into the vacuum towards the tip region varies when a sharp tip is considered. This will cause modifications in the coupling of high FER states to bulk right electrode states.

\section{CONCLUSIONS}

In this work, a novel approach to investigate FERs is introduced. Special emphasis has been placed on the selfconsistent treatment of the finite bias nonequilibrium problem in tunneling regime and on the effect of the image potential. A precise simulation of FERs is possible thanks to the use of a transport calculation method based on the use of a plane wave basis set. Such a basis set allows numerically accurate calculations for the large vacuum space in the description of FERs.

Using this approach, we have simulated FERs of the $\mathrm{Cu}(100)$ surface. The constant current operation mode has been mimicked by calculating FERs for increasing values of the vacuum gap distance and of the applied bias voltage. In this way, we have been able to reproduce FERs with different quantum numbers up to $n=4$. The role of the image potential is found to be essential for low quantum number FERs, whereas for high quantum numbers, the FERs can be influenced by the tip induced electric field. We have also shown that within the range of bias voltages used in STS experiments, a self-consistent treatment of the problem is desirable.

A practical strategy has been followed to simulate inelastic transmission through FERs, giving rise to differential conductance spectra with sharp peaks at the position of FERs. Furthermore, our procedure incorporates a full threedimensional treatment of the surface potential, for both tip and sample. Hence it can be applied to any tip shape and permits exploring tip effects on FERs, which can be of great interest for the interpretation of experimental observations.

\section{ACKNOWLEDGMENTS}

We acknowledge support from the Basque Departamento de Educación and the UPV/EHU (Grant No. IT-756-13), the Spanish Ministerio de Ciencia e Innovacióon (Grant No. FIS2010-19609-C02-00), and the ETORTEK program funded by the Basque Departamento de Industria and the Diputación Foral de Gipuzkoa. It used the resources of the National Energy Research Scientific Computing Center (NERSC). L.W. Wang is supported by the US DOE/SC/BES under contract No. DE-AC02-05CH11231.
${ }^{1}$ H. Nienhaus, Surf. Sci. Rep. 45, 1 (2002).

${ }^{2}$ P. M. Echenique, R. Berndt, E. V. Chulkov, T. Fauster, A. Goldmann, and U. Höfer, Surf. Sci. Rep. 52, 219 (2004).

${ }^{3}$ P. Echenique and J. Pendry, Prog. Surf. Sci. 32, 111 (1989).

${ }^{4}$ N. Smith, Phys. Rev. B 32, 3549 (1985).
${ }^{5}$ N. Smith, Rep. Prog. Phys. 51, 1227 (1988).

${ }^{6} \mathrm{R}$. Wiesendanger, Scanning Probe Microscopy and Spectroscopy: Methods and Applications (Cambridge University Press, Cambridge, 1994).

${ }^{7}$ R. Shantyr, Ph.D. thesis, Martin-Luther-Universität HalleWittenberg, 2004. 
${ }^{8}$ T. Jung, Y. W. Mo, and F. J. Himpsel, Phys. Rev. Lett. 74, 1641 (1995).

${ }^{9}$ M. Pivetta, F. Patthey, M. Stengel, A. Baldereschi, and W. D. Schneider, Phys. Rev. B 72, 115404 (2005).

${ }^{10}$ J. A. Kubby, Y. R. Wang, and W. J. Greene, Phys. Rev. Lett. 65, 2165 (1990).

${ }^{11}$ A. J. Caamaño, Y. Pogorelov, O. Custance, J. Mendez, M. A. Baró, J. Y. Veuillen, J. Gómez-Rodríguez, and J. J. Sáenz, Surf. Sci. 426, L420 (1999).

${ }^{12}$ K. Bobrov, A. J. Mayne, and G. Dujardin, Nature (London) 413, 616 (2001).

${ }^{13}$ A. Kubetzka, M. Bode, and R. Wiesendanger, Appl. Phys. Lett. 91, 012508 (2007).

${ }^{14}$ J. I. Pascual, C. Corriol, G. Ceballos, I. Aldazabal, H.-P. Rust, K. Horn, J. M. Pitarke, P. M. Echenique, and A. Arnau, Phys. Rev. B 75, 165326 (2007).

${ }^{15}$ D. B. Dougherty, P. Maksymovych, J. Lee, M. Feng, H. Petek, and J. J. T. Yates, Phys. Rev. B 76, 125428 (2007).

${ }^{16}$ P. Wahl, M. A. Schneider, L. Diekhöner, R. Vogelgesang, and K. Kern, Phys. Rev. Lett. 91, 106802 (2003).

${ }^{17}$ A. Pronschinske and D. B. Dougherty, J. Phys. Chem. Lett. 1, 2613 (2010).

${ }^{18}$ K. Schouteden and C. Van Haesendonck, Phys. Rev. Lett. 108, 076806 (2012).

${ }^{19}$ S. Stepanow, A. Mugarza, G. Ceballos, P. Gambardella, I. Aldazabal, A. G. Borisov, and A. Arnau, Phys. Rev. B 83, 115101 (2011).

${ }^{20}$ J. M. Pitarke, F. Flores, and P. M. Echenique, Surf. Sci. 234, 1 (1990).

${ }^{21}$ G. Butti, S. Caravati, G. P. Brivio, M. I. Trioni, and H. Ishida, Phys. Rev. B 72, 125402 (2005).

${ }^{22}$ S. Crampin, Phys. Rev. Lett. 95, 046801 (2005).

${ }^{23}$ A. Hanuschkin, D. Wortmann, and S. Blügel, Phys. Rev. B 76, 165417 (2007).

${ }^{24}$ A. G. Eguiluz, M. Heinrichsmeier, A. Fleszar, and W. Hanke, Phys. Rev. Lett. 68, 1359 (1992).

${ }^{25}$ G. Fratesi, G. P. Brivio, P. Rinke, and R. W. Godby, Phys. Rev. B 68, 195404 (2003).

${ }^{26}$ M. Nekovee and J. Inglesfield, Europhys. Lett. 19, 535 (1992).

${ }^{27}$ P. Echenique and J. Pendry, J. Phys. C 11, 2065 (1978).

${ }^{28}$ R. O. Jones, P. J. Jennings, and O. Jepsen, Phys. Rev. B 29, 6474 (1984).
${ }^{29}$ L. W. Wang, Phys. Rev. B 72, 045417 (2005).

${ }^{30}$ A. Garcia-Lekue and L. W. Wang, Phys. Rev. B 74, 245404 (2006).

${ }^{31}$ M. G. Vergniory, C. Yang, A. Garcia-Lekue, and L. Wang, Comp. Mat. Sci. 48, 544 (2010).

${ }^{32}$ A. Garcia-Lekue and L. W. Wang, Phys. Rev. B 82, 035410 (2010).

${ }^{33}$ A. Zugarramurdi, N. Zabala, A. G. Borisov, and E. V. Chulkov, Phys. Rev. B 84, 115422 (2011).

${ }^{34}$ A. Pronschinske, D. J. Mardit, and D. B. Dougherty, Phys. Rev. B 84, 205427 (2011)

${ }^{35}$ E. V. Chulkov and V. M. Silkin, Surf. Sci. 437, 330 (1999).

${ }^{36}$ W. Berthold, U. Höfer, P. Feulner, E. V. Chulkov, V. M. Silkin, and P. M. Echenique, Phys. Rev. Lett. 88, 056805 (2002).

${ }^{37}$ A. Garcia-Lekue and L. W. Wang, Comp. Mat. Sci. 45, 1016 (2009),

${ }^{38}$ X. W. Jiang, S. S. Li, J. B. Xia, and L. W. Wang, J. Appl. Phys. 109, 054503 (2011)

${ }^{39}$ N. D. Lang and W. Kohn, Phys. Rev. B 7, 3541 (1973).

${ }^{40}$ E. V. Chulkov, V. M. Silkin, and P. M. Echenique, Surf. Sci. 391, L1217 (1997).

${ }^{41}$ G. Binnig, N. García, H. Rohrer, J. M. Soler, and F. Flores, Phys. Rev. B 30, 4816 (1984).

${ }^{42}$ J. B. Pendry, Low Energy Electron Diffraction (Academic Press Inc., London, 1974).

${ }^{43}$ R. García, J. J. Sáenz, J. M. Soler, and N. García, Surf. Sci. 181, 69 (1987).

${ }^{44}$ T. Fauster and W. Steinmann, in Electromagnetic waves: Recent Developments in Research, edited by P. Halevi (North-Holland, Amsterdam, 1995), p. 350.

${ }^{45}$ M. Machado, E. V. Chulkov, V. M. Silkin, U. Höfer, and P. M. Echenique, Prog. Surf. Sci. 74, 219 (2003).

${ }^{46}$ From the full system potential (SC or non-SC) and following the procedure described in Refs. 29 and 30, one can easily obtain the electronic states of the full system. Then, the FERs are easily identified by searching for those states that are nondegenerate and are confined near the surface in the vacuum region.

${ }^{47}$ D. Straub and F. J. Himpsel, Phys. Rev. Lett. 52, 1922 (1984).

${ }^{48}$ K. Giesen, F. Hage, F. J. Himpsel, H. J. Riess, and W. Steinmann, Phys. Rev. B 35, 971 (1987).

${ }^{49}$ B. Borca, S. Barja, M. Garnica, D. Sánchez-Portal, V. M. Silkin, E. V. Chulkov, C. F. Hermanns, J. J. Hinarejos, A. L. Vázquez de Parga, A. Arnau, P. M. Echenique, and R. Miranda, Phys. Rev. Lett. 105, 036804 (2010).

${ }^{50} \mathrm{C}$. Corriol (private communication). 